\title{
SHORT COMMUNICATION Direction-specific motion blindness induced by focal stimulation of human extrastriate cortex
}

\author{
O. Blanke, ${ }^{1,3}$ T. Landis, ${ }^{1}$ A. B. Safran ${ }^{2}$ and M. Seeck ${ }^{1,3}$ \\ ${ }^{1}$ Department of Neurology, University Hospital of Geneva, 24 rue Micheli-du-Crest, 1211 Geneva, Switzerland \\ ${ }^{2}$ Department of Ophthalmology, Geneva University Hospitals, Geneva, Switzerland \\ ${ }^{3}$ Functional Neurosurgery and Neurology program of the Universities of Lausanne and Geneva, Switzerland
}

\begin{abstract}
Motion blindness (MB) or akinetopsia is the selective disturbance of visual motion perception while other features of the visual scene such as colour and shape are normally perceived. Chronic and transient forms of MB are characterized by a global deficit of direction discrimination (pandirectional), which is generally assumed to result from damage to, or interference with, the motion complex MT+/V5. However, the most characteristic feature of primate MT-neurons is not their motion specificity, but their preference for one direction of motion (direction specificity). Here, we report that focal electrical stimulation in the human posterior temporal lobe selectively impaired the perception of motion in one direction while the perception of motion in other directions was completely normal (unidirectional MB). In addition, the direction of MB was found to depend on the brain area stimulated. It is argued that direction specificity for visual motion is not only represented at the single neuron level, but also in much larger cortical units.
\end{abstract}

Keywords: motion perception, motion blindness, human, electrical stimulation, direction selectivity, extrastriate cortex

\section{Introduction}

Some patients with posterior brain damage rarely suffer from a selectively weakened capacity to perceive visual motion (Zihl et al., 1983; Vaina, 1989; Zeki, 1991). This phenomenon has become known as motion blindness (MB) and usually follows extensive and bilateral posterior brain damage. Yet, less severe MB has also been described following unilateral posterior brain lesions (Plant et al., 1993; Barton et al., 1995; Greenlee \& Smith, 1997; Schenk \& Zihl, 1997; Vaina et al., 2001) and transcranial magnetic stimulation (TMS) over extrastriate cortex in healthy subjects (Bekkers \& Hömberg, 1992; Hotson et al., 1994; Pascual-Leone \& Walsh, 2001). MB has also been produced experimentally in the macaque subsequent to unilateral (Newsome \& Paré, 1988; Rudolph \& Pasternak, 1999) or bilateral ablation of area MT/V5 (Pasternak \& Merigan, 1994). Area MT/V5 was chosen by the latter authors as it contains a large number of direction selective neurons (Dubner \& Zeki, 1971; Zeki, 1974; Van Essen et al., 1981) that are organized in columns of cells tuned to similar directions of motion (Albright et al., 1984). Based on these animal data as well as lesion overlap in humans (Barton et al., 1995; Vaina et al., 2001), MB is generally supposed to result from damage to direction selective neurons in human area $\mathrm{MT}+/ \mathrm{V} 5$, which is believed to be homologueous to macaque areas MT and MST (e.g. Zihl et al., 1983; Zeki, 1991; Bekkers \& Hömberg, 1992; Pascual-Leone \& Walsh, 2001).

MB in both species is generally described as a global deficit of motion direction discrimination that is similar in strength for all tested directions (pandirectional MB; Zihl et al. 1983; Zeki, 1991;

Correspondence: Dr O. Blanke, as above.

E-mail: olaf.blanke@hcuge.ch

Received 5 February 2002, revised 17 March 2002, accepted 23 March 2002
Bekkers \& Hömberg, 1992; Hotson et al., 1994). However, the most characteristic feature of neurons in area MT/V5 is not their motion specificity, but their preference for one direction of motion (direction specificity; Dubner \& Zeki, 1971; Zeki, 1974; Van Essen et al., 1981; Albright et al., 1984). It might thus be conjectured that focal interference with human area MT+/V5 (by brain damage or TMS) might not only lead to a motion specific visual discrimination deficit (pandirectional $\mathrm{MB}$ ) as generally stated, but to a direction-specific impairment of motion perception. To investigate whether unidirectional $\mathrm{MB}$, and thus direction specificity, occurs in human extrastriate cortex, the effect of focal electrical stimulation (ES) on visual motion discrimination was tested in a patient undergoing invasive monitoring for epilepsy.

\section{Materials and methods}

Patient

This 43-year-old right-handed female patient had suffered from epilepsy since she was 32 years old. Her complex partial seizures started with an epigastric aura followed by the sensation of globally diminished hearing. These manifestations were followed by starring, loss of consciousness and bimanual automatisms. There were no secondary generalizations and no postictal aphasia. Although she rarely experienced visual psychic auras (characterized by the 'dreamlike' impression of 'seeing an image of herself'), her seizures were never accompanied by other visual phenomena and, in particular, never by the perception of visual motion. Pharmacoresistancy was diagnosed and she was addressed to noninvasive presurgical epilepsy evaluation, which consisted of ictal and interictal video-EEG recordings, three dimensional magnetic 


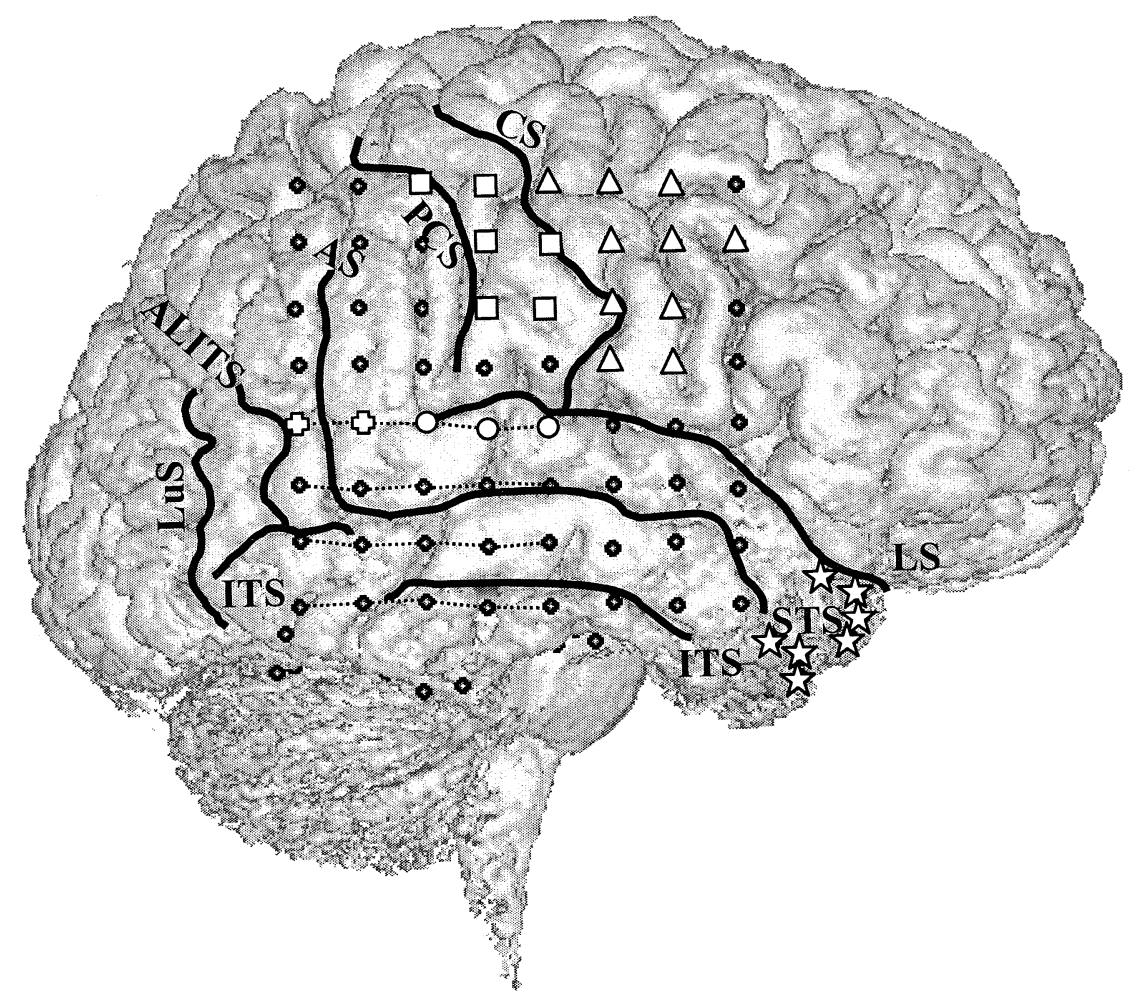

FIG. 1. Anatomical location of sites inducing motion blindness and other behavioural responses. Magnetic resonance imaging 3D surface reconstruction of the right hemisphere of patient FM depicting sites where electrical stimulation (ES) evoked behavioural responses. Motion direction discrimination was tested in the area indicated by black (dashed) lines between adjacent electrodes. Primary sensory and motor responses, as well as major sulci are indicated. Note that the epileptic focus (indicated by the stars) was found in the anterior and medial temporal lobe, and thus $>3 \mathrm{~cm}$ in front of sites where motion direction discrimination was investigated (not all medial temporal sites are shown). Key to symbols and abbreviations: triangle, motor response; square, somatosensory response; circle, auditory response; cross, vestibular response; star, epileptic focus; black dots, no overt response. CS, central sulcus; LS, lateral sulcus; STS, superior temporal sulcus; PCS, postcentral sulcus; ITS, inferior temporal sulcus; ALITS, ascending limb of the inferior temporal sulcus; AS, angular sulcus; LuS, lunate sulcus. The anatomical location of area MT+/V5 is at the intersection the posterior ITS and the ALITS (Dumoulin et al., 2000).

resonance imaging (MRI), interictal positron emission tomography, interictal and ictal single photon emission computer tomography, and interictal and postictal neuropsychological examinations. The neurological examination was normal, as were the visual fields of both eyes (Goldmann dynamic perimetry). Based on these examinations, right temporal lobe epilepsy was diagnosed. However, as 3D-MRI did not reveal any lesion, invasive monitoring was indicated to localize the seizure focus more precisely. Subdural grid electrodes (Ad-Tech Corp., Racine, WI, USA) were implanted to record seizures, and electrical stimulation was performed to plan the surgical resection. Informed written consent was obtained and ES was conducted in conformity with the Declaration of Helsinki. The invasive procedure confined the seizure onset to the right amygdala and the immediately surrounding cortex (Fig. 1); seizure onset was followed by ictal propagation to more posterior temporal and parietal regions. Right anterior temporal lobectomy was performed and the patient has been seizure free for 12 months.

\section{Invasive recordings and electrical cortical stimulation}

Subdural grid electrodes were 3-mm-diameter stainless-steel electrodes with a centre-to-centre distance of $8 \mathrm{~mm}$. Subdural electrodes were MRI-compatible and embedded in a clear silastic sheet. Electrode location was determined by intraoperative photographs and 3-D MRI of the brain with the implanted electrodes as described previously (Blanke et al., 2000). A total of 88 subdural grid electrodes were placed over the right hemisphere covering the lateral temporal lobe and also parts of the basal temporal lobe, the parietal lobe and the frontal lobe (see Fig. 1; not all basal temporal and anterior temporal strip electrodes are shown). ES was performed with a Grass Stimulator S12 (Grass Instruments, Quincy, MA, USA) and enabled localization of primary motor and somatosensory cortex as well as auditory and vestibular cortices (Fig. 1). Constant-current ES (amplitude, 1-15 mA; biphasic regular pulses with pulse duration, $0.3 \mathrm{~ms}$; train duration, $2 \mathrm{~s}$ ), which was below the threshold of afterdischarges, was applied at $50 \mathrm{~Hz}$ in a bipolar fashion through horizontally adjacent contacts on the cortical surface (Lesser et al., 1987; Blanke et al., 2000). During bipolar stimulation patient FM performed a variety of tasks including naming, reading, writing, counting and neglect testing in accordance with Lesser et al. (1987).

\section{Stimuli and apparatus}

Visual motion discrimination was tested at 16 cortical sites in the right posterior temporal lobe (see Fig. 1) using a random dot display on a computer screen and a five-alternative forced choice discrimination task (verbal responses: 'right'; 'left'; 'up'; 'down'; or 'not perceived', Losey et al., 1998). Coherent motion stimuli (duration, $2 \mathrm{~s}$ ) were presented synchronously with ES on a 20-inch computer monitor (Sony; frame rate, $70 \mathrm{~Hz} ; 640 \times 480$ pixels) in black and white in a normally lit room (Losey et al., 1998). Viewing distance was $100 \mathrm{~cm}$. The stimuli were presented in a borderless square of $12^{\circ} \times 12^{\circ}$ in the left (contralateral) visual field (inner border was $20^{\circ}$ left of the fixation spot). This was done to activate mainly right area 

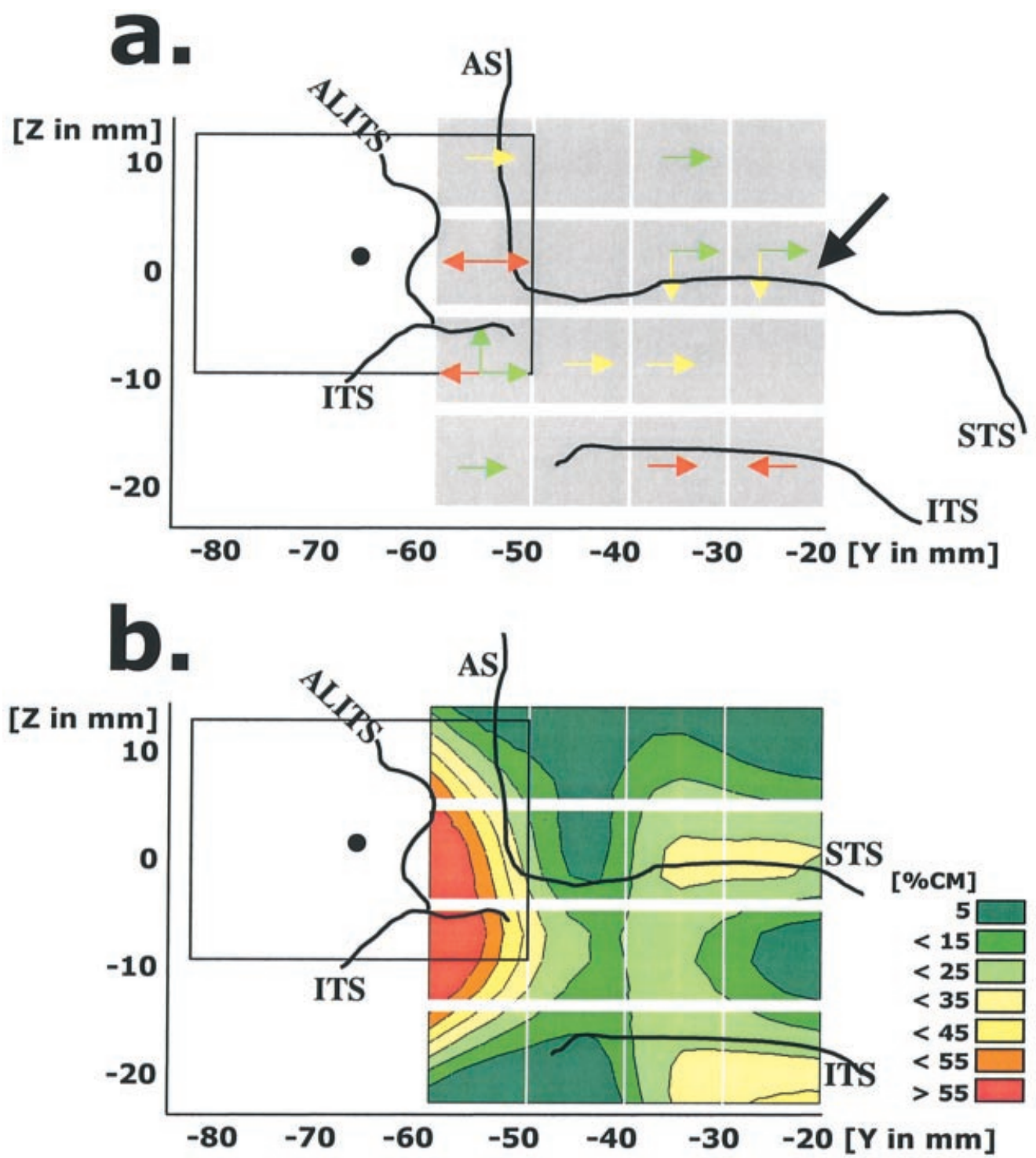

FIG. 2. (a)Direction of motion blindness. This panel depicts the direction(s) of motion that were selectively impaired by electrical stimulation (ES) during the direction discrimination task. For each of the 16 tested sites the direction and strength of MB in the contralateral visual field is indicated. As ES was bipolar, the 20 electrodes shown in Fig. 1 correspond to 16 bipolar stimulation sites. (A grey square depicts one bipolar stimulation site.) The direction of the arrow indicates the direction of MB $(\rightarrow$, ipsilateral; $\leftarrow$ contralateral; $\uparrow$, up; $\downarrow$, down). The colour code for the strength of MB is defined as follows. The colour of the arrow indicates the threshold for motion discrimination during ES: green, $>44.4 \% \mathrm{CM}$; yellow, $>66.7 \% \mathrm{CM}$; red, $>100 \% \mathrm{CM}$, black, normal threshold (44.4\% CM correctly discriminated). The stereotaxic location ( $y$ and $z$ coordinates) of area MT+/V5 is indicated by its mean (black dot) \pm 1 standard deviation (black frame; Watson et al., 1993). Note that the two sites located in the lower and anterior part of the stereotaxic borders of area MT+/V5 show the most global or pandirectional MB. Major sulci (AS, ITS, ST and ALITS) are also indicated for orientation (see Fig. 1). Abbreviations are as in Fig. 1. (b)Strength of motion blindness. The predominant implication of area MT+/V5 in motion direction discrimination is highlighted. The same 16 bipolar sites as Fig. 2a are depicted in their stereotaxic location. Here, the strength of MB in the contralateral visual field, calculated as the mean threshold of all tested directions at each site, is shown for each site (see methods). A least square fit was chosen to indicate the strength of MB over the tested cortex. Note that sites located within the stereotaxic borders of area MT+/V5 show strongest MB (indicated in red). Ordinate and abscissa as in Fig. 2a. Note that ES of two areas anterior to MT+/V5, one on the STS and the other on the ITS (each consisting of two bipolar stimulation sites), also leads to prominent MB. Both areas are indicated in yellow depicting an intermediate average discrimination threshold. Major sulci (AS, ITS, ST and ALITS) are again indicated for orientation (see Fig. 1). Abbreviations are as in Fig. 2a.

$\mathrm{MT}+/ \mathrm{V} 5$, as receptive fields in this area not only cover the contralateral visual field, but extend up to $20^{\circ}$ into the ipsilateral visual field. This has been shown in man (Tootell et al., 1995) and monkey (Van Essen et al., 1981). If a motion direction discrimination deficit was found in the contralateral visual field, impaired directions of motion were also tested in the ipsilateral visual field. During the stimulation, eye movements were monitored by direct inspection and video recordings. Trials with eye movements during stimulus presentation were excluded from analysis. Each random dot field contained 1000 dots (diameter, $0.29^{\circ}$ ). The dots had a random duration between 4 and $800 \mathrm{~ms}$ and were randomly positioned within the square. A percentage of the dots was programmed to be displaced with a velocity of $2^{\circ}$ per second in the tested direction (signal dots). The coherence of motion $(\mathrm{CM})$ was expressed as a percentage, defined as $100 \times$ (the number of signal dots/the total number of dots). Prior to the trials with ES, direction discrimination was 
measured by determining the minimum percentage of dots moving coherently in one of the four cardinal directions (right, left, up or down) that was correctly discriminated in all test trials (absolute discrimination threshold). An absolute discrimination threshold was defined at $\mathrm{CM}=44.4 \%$ for all four directions and both visual fields. Although on some trials (without ES) the patient discriminated motion stimuli at 29.6 or $19.7 \% \mathrm{CM}$, her performance at these levels of CM was too variable to be used during ES. Thus, in accordance with protocols that are used to determine stimulation-induced language impairments (or other cognitive deficits) in epileptic patients during presurgical workup (i.e. Lesser et al., 1987), we investigated the effects of ES on motion direction discrimination at the absolute discrimination threshold. This was done in order to be as certain as possible that potential threshold elevations during ES were evoked by electrical interference and not related to variability of performance. It also enabled us to minimize the number of ES trials, which needed to be as low as possible due to the clinical situation (total of 115 trials; $7.2 \pm 2.0$ trials per stimulation site; mean $\pm \mathrm{SD}$ ). During ES, three stimuli with different percentages of correlated dots at and above the absolute discrimination threshold (CM: 44.4, 66.7 or $100 \%$ ) could be presented for each direction. Motion direction was varied pseudorandomly in trials with and without ES in the contralateral visual field. Initial stimulus presentation at each site was always given at $\mathrm{CM}=44.4 \%$. If a motion stimulus in a given direction was correctly discriminated at $\mathrm{CM}=44.4 \%$, this direction was not retested at higher threshold values. A motion discrimination deficit in a given direction was defined as an increase in threshold, ie. $\mathrm{CM}>44.4 \%$, which had to be confirmed in at least one other trial. The absolute discrimination threshold in a healthy control group ( $n=9$; 3 women and 6 men; mean age $36.6 \pm 8.5$ years; 8 righthanded, 1 ambidextrous; all without neurological or ophthalmological disorders) was lower and found at $\mathrm{CM}=13.4 \pm 5.1 \%$ (for both visual fields), which might be due to antiepileptic medication, craniotomy and the clinical situation of stimulation mapping in the present patient. Flawless performance at $\mathrm{CM}=44.4 \%$ was confirmed in further control trials. Minimal duration between trials with ES was $>15 \mathrm{~s}$. All the tests for motion discrimination were carried out on the same day. The global strength of MB was calculated for each site. This was done by calculating the average motion discrimination threshold of the four tested directions for each site (mean of percentage CM for right, left, up and down).

\section{Results}

Motion direction discrimination in the contralateral visual field was tested at 16 bipolar stimulation sites. At seven sites motion discrimination was impaired in the contralateral visual field (increase of threshold to $\mathrm{CM}>44.4 \%$ is indicated by green arrows, and to $\mathrm{CM}>66.7 \% \mathrm{CM}$ by yellow arrows, see Fig. 2a) and at four further sites completely extinguished (even at $\mathrm{CM}=100 \%$ motion was not perceived; red arrows, Fig. 2a). MB was unidirectional at seven sites, bidirectional at three sites and tridirectional at one site (Fig. 2a, direction of arrow indicates direction of MB). Motion discrimination was most often weakened in the ipsilateral direction (with respect to stimulated hemisphere; arrow pointing to the right in Fig. 2a) accounting for $63 \%$ of all deficits. Other deficits were: contralateral $(19 \%$, left), down $(13 \%)$ and up $(5 \%)$.

Qualitatively, ES changed the experience of directional motion stimuli to one of random motion. ES did not induce the experience of zigzag motion, blurred motion, curved motion or phosphenes, as commonly induced by extracranial TMS of extrastriate cortex
(Bekkers \& Hömberg, 1992; Hotson et al., 1994; Pascual-Leone \& Walsh, 2001). Instead of experiencing such an addition of motion to the presented motion stimulus, the patient reported that the presented motion stimulus was weakened. She compared it to directed motion stimuli at $\mathrm{CM}=8.8$ or $13.2 \%$ that were below the discrimination threshold without ES. This qualitative effect was found at all sites that induced MB. Most importantly, this loss of directional motion perception was only found for the direction(s) that could not be discriminated anymore. For example, ES at the site that is indicated by a black arrow (most anterior stimulation site just above the superior temporal sulcus) in Fig. 2a weakened the perception of motion going in the ipsilesional and down direction to random motion (ipsilesional and downward MB). The application of the same current at the same site did not modify the experience of a stimulus with the same percentage of signal dots going upwards or contralesional. ES without stimulus presentation did not lead to any reportable phenomena.

$\mathrm{MB}$ was found to be specific for the contralateral visual field, as motion direction discrimination in the ipsilateral visual field was flawless during ES. This was tested at seven of 11 sites that induced MB (total number of trials, 20) and showed that the direction of motion that was not perceived in the contralateral visual field was normally perceived in the ipsilateral visual field. Motion direction discrimination in the ipsilateral visual field was not retested for directions that were normally perceived in the contralateral visual. Currents applied during the presentation of contralateral motion stimuli were constant within and across sites (mean, $4.0 \pm 1.2 \mathrm{~mA}$ ) and even lower than those applied during ipsilateral stimulus presentation (range, 8.0-14.0 mA). Direction specific MB was not related to left-sided hemianopia, visual field defects or impaired contrast thresholds, as a global motion deficit should have resulted in this case. Secondly, impaired contrast thresholds should also impair line orientation judgements, which was not the case (tested at all sites that induced MB). We also excluded stimulation-induced left-sided neglect by normal line bisection and extinction testing.

$\mathrm{MB}$ was found at sites in the superior, middle and inferior temporal gyrus as well as the angular gyrus. The direction of MB was found to depend on the cortical site stimulated. Thus, in the investigated area the predominant direction of $\mathrm{MB}$ varied and was either ipsilateral, contralateral or downward. However, ES at remote bipolar sites on different gyri can lead to ipsidirectional MB, which is identical in strength (e.g. leftward MB on the middle part of inferior temporal sulcus and on the posterior part of the middle temporal sulcus, Fig. 2a). Although not determined statistically, the strongest MB (as defined by the average direction discrimination threshold) and most global MB (bi- and tridirectional deficit) was induced on the cortex anterior to the ascending limb of the inferior temporal sulcus (two posterior red sites, see Fig. 2a and b), which has been defined as the anatomical landmark of area MT+/V5 (Dumoulin et al., 2000). The Talairach coordinates of these two sites $(x, y, z=60,-54,2 ; 60,-53$, -9) are also within the proposed stereotaxic location [mean (black dot) \pm SD (black box) in Fig. 2a and b] of area MT+/V5 as proposed by Watson et al. (1993).

\section{Discussion}

The present observations demonstrate that unilateral ES of discrete sites in extrastriate cortex specifically diminished or extinguished the capacity to discriminate motion in the contralateral visual field. Intracranial stimulation thus reproduced transient $\mathrm{MB}$ as described in patients with unilateral posterior brain damage (Barton et al., 1995; 
Greenlee \& Smith, 1997; Schenk \& Zihl, 1997; Vaina et al., 2001) or induced by TMS in normal subjects (Bekkers \& Hömberg, 1992; Hotson et al., 1994). Most importantly however, the visual deficit in patient FM was not only motion specific as previously reported, but also direction specific. This impairment in performance was accompanied by the stimulation-induced experience of a strongly weakened motion signal for the directions that could not be discriminated anymore. Unimpaired directions were unmodified and experienced in the same way with and without ES. Based on these quantitative and qualitative data, it is argued that direction-specific MB resulted from a stimulation-induced transient dysfunction of direction selective neurons in the cortical motion pathway. An important implication of this pathway and especially area MT/V5 in the perception of the direction of motion has been demonstrated by single unit recordings (Dubner \& Zeki, 1971; Zeki, 1974; Van Essen et al., 1981; Albright et al., 1984), microstimulation (Salzman et al., 1990, 1992), and circumscribed lesions (Newsome \& Paré, 1988; Rudolph \& Pasternak, 1999). The present results suggest that direction-specific MB at a given site might have resulted from selective or predominant dysfunction of neurons in the cortical motion pathway tuned to the direction of motion that patient FM did not discriminate anymore. Neurons encoding other directions of motion might have been less impaired or unimpaired leading to normal motion vision in those directions. As the electrical currents in the present study were applied to a large area (as compared to microstimulation in monkey; Salzman et al., 1992), the present data suggest that direction-of-motion specificity might not only be carried at the single cell level, or in neuronal clusters as suggested by Malonek et al. (1994), but also in larger motion-processing units.

Although prominent $\mathrm{MB}$ (motion not discriminated at $\mathrm{CM}=100 \%$ ) was induced at several sites in the posterior temporal lobe, the present results are concordant with the predominant role of area $\mathrm{MT}+/ \mathrm{V} 5$ in visual motion processing. Thus, ES at sites in area MT+/V5 (Watson et al., 1993; Dumoulin et al., 2000) induced the strongest and most global MB (see Fig. 2a and b). Given the fact that area MT+/V5 is generally buried within the ascending limb of the inferior temporal sulcus and that its mean stereotaxic position lies just posterior to this sulcus, it seems possible that ES at these latter sites might lead to greater, and even pandirectional, MB. ES of two areas anterior to area MT+/V5 also led to prominent, although less strong MB than ES of MT+/V5. One area was localized at the superior temporal sulcus and the other at the inferior temporal sulcus. Recent neuroimaging evidence suggests the presence of highly specialized motion areas in both regions. It might be proposed that the area at the superior temporal sulcus might be concordant with an area that analyses body motion (Puce et al., 1998) and that the area at the inferior temporal sulcus is concordant with an area that is specialized for object motion (Beauchamp et al., 2002).

The specificity and redundancy of direction-specific MB in patient FM suggests that each motion area is necessary, but not sufficient, for the perception of motion direction. This conclusion, however, must be regarded with caution for two reasons. First, it can be argued that our results cannot be applied to normal brain function, as ES has been carried out in a patient with right temporal lobe epilepsy. However, patient FM's epileptic focus was found in the anterior medial temporal lobe and the location of sensory and motor functions did not suggest deviant brain pathology with respect to anatomical representation of cortical functions. Second, direction-specific MB might not only result from impaired motion processing in the directly stimulated area of the motion network but also from more distant effects via stimulation of passing fibers and transynaptic current spread (Salzman et al., 1992). Yet, the direction specificity of MB at each site indicates that the induced currents mainly spread to neuronal populations with similar directional properties.

To conclude, the present observations suggest that single directions of visual motion are represented in a number of extrastriate areas at a macroscopic level, and that normal processing in the entire extrastriate motion network is necessary for correct direction-ofmotion perception.

\section{Acknowledgements}

This work was supported by grants from the Swiss National Science Foundation (3100-67874.02, 3100-065232.01, 3100-067105.01). We thank S. Ortigue, L. Spinelli, C. Mermoud and S. Perrig for technical help and M. Seghier for critical comments on an earlier version of the manuscript.

\section{Abbreviation}

$\mathrm{CM}$, coherence of motion.

\section{References}

Albright, T.D., Desimone, R. \& Gross, C.G. (1984) Columnar organization of directionally selective cells in visual area MT of the macaque. $J$. Neurophysiol., 51, 16-31.

Barton, J.S., Sharpe, J.A. \& Raymond, J.E. (1995) Retinotopic and directional defects in motion discrimination in humans with cerebral lesions. Ann. Neurol., 37, 665-675.

Beauchamp, M.S., Lee, K.E., Haxby, J.V. \& Martin, A. (2002) Parallel visual motion processing streams for manipulable objects and human movements. Neuron, 34, 49-159.

Bekkers, G. \& Hömberg, V. (1992) Cerebral visual motion blindness: transitory akinetopsia induced by transcranial magnetic stimulation of human area V5. Proc. R. Soc. Lond. B, 249, 173-178.

Blanke, O., Spinelli, L., Thut, G., Michel, C.M., Perrig, S., Landis, T. \& Seeck, M. (2000) Location of the human frontal eye field as defined by electrical cortical stimulation: anatomical, functional and electrophysiological characteristics. Neuroreport, 11, 1907-1913.

Dubner, R. \& Zeki, S.M. (1971) Response properties and receptive fields of an anatomically defined region of the superior temporal sulcus in the monkey. Brain Res., 35, 528-532.

Dumoulin, S.O., Bittar, R.G., Kabani, N.J., Baker, C.L. Jr, Le Goualher, G., Bruce, P.G. \& Evans, A.C. (2000) A new anatomical landmark for reliable identification of human area V5/MT: a quantitative analysis of sulcal patterning. Cereb. Cortex, 10, 454-463.

Greenlee, M.W. \& Smith, A.T. (1997) Detection and discrimination of first and second order motion in patients with unilateral posterior brain damage. J. Neurosci., 17, 804-818.

Hotson, J., Braun, D., Herzberg, W. \& Boman, D. (1994) Transcranial magnetic stimulation of extrastriate cortex degrades human motion direction discrimination. Vision Res., 34, 2115-2123.

Lesser, R.P., Luders, H., Klem, G., Dinner, D.S., Morris, H.H., Hahn, J.F. \& Wyllie, E. (1987) Extraoperative cortical functional localization in patients with epilepsy. J. Clin. Neurophysiol., 4, 27-53.

Losey, F., Safran, A.B., Michel, C.M. \& Landis, T. (1998) Perception visuelle du mouvement. Etude normative. Klin. Monatsbl. Augenheilkd., 212, 379381

Malonek, D., Tootell, R.B. \& Grinvald, A. (1994) Optical imaging reveals the functional architecture of neurons processing shape and motion in owl monkey area MT. Proc. R. Soc. Lond. B, 258, 109-119.

Newsome, W.T. \& Paré, E.B. (1988) A selective impairment of motion perception following lesions to the middle temporal visual area (MT). $J$. Neurosci., 8, 2201-2211.

Pascual-Leone, A. \& Walsh, V. (2001) Fast backprojections from the motion to the primary visual area necessary for visual awareness. Science, 292, 510-512.

Pasternak, T. \& Merigan, W.H. (1994) Motion perception following lesions of the superior temporal sulcus in the monkey Cereb. Cortex, 4, 247-259.

Plant, G.T., Laxer, K.D., Barbaro, N.M., Schiffman, J.S. \& Nakayama, K. (1993) Impaired visual motion processing in the contralateral hemifield 
following unilateral posterior cerebral lesions in humans. Brain, 116, 1303 1335.

Puce, A., Allison, T., Bentin, S., Gore, J.C. \& McCarthy, G. (1998) Temporal cortex activation in humans viewing eye and mouth movements. $J$. Neurosci., 18, 2188-2199.

Rudolph, K. \& Pasternak, T. (1999) Transient and permanent deficits in motion perception after lesions of cortical areas MT and MST in the macaque monkey. Cereb. Cortex, 9, 90-100.

Salzman, C.D., Britten, K.H. \& Newsome, W.T. (1990) Cortical microstimulation influences perceptual judgements of motion direction. Nature, 346, 174-177.

Salzman, C.D., Murasugi, C.M., Britten, K.H. \& Newsome, W.T. (1992) Microstimulation in visual area MT: effects on direction discrimination performance. J. Neurosci., 12, 2331-2355.

Schenk, T. \& Zihl, J. (1997) Deficits in global motion perception. Neuropsychologia, 35, 1289-1297.

Tootell, R.B.H., Reppas, J.B., Kwong, K.K., Malach, R., Born, R.T., Brady, T.J., Rosen, B.R. \& Belliveau, J.W. (1995) Functional analysis of human MT and related visual cortical areas using magnetic resonance imaging. $J$. Neurosci., 15, 3215-3230.
Vaina, L.M. (1989) Selective impairment of visual motion interpretation following lesions of the right occipito-parietal area in humans. Biol. Cybern., 61, 347-359.

Vaina, L.M., Cowey, A., Eskew, R.T., LeMay, M. \& Kemper, T. (2001) Regional cerebral correlates of global motion perception: evidence from unilateral cerebral brain damage. Brain, 124, 310-321.

Van Essen, D.C., Maunsell, J.H.R. \& Bixby, J.L. (1981) The middle temporal visual area in the macaque: myeloarchitecture, connections, functional properties and topographic organization. J. Comp. Neurol., 199, 293-326.

Watson, J.D.G., Watson, J.D., Myers, R., Frackowiak, R.S., Hajnal, J.V., Woods, R.P., Mazziotta, J.C., Shipp, S. \& Zeki, S.M. (1993) Area V5 of the human brain: evidence from a combined study using positron emission tomography and magnetic resonance imaging. Cereb. Cortex, 3, 79-94.

Zeki, S.M. (1974) Functional organization of a visual area in the posterior bank of the superior temporal sulcus of the rhesus monkey. J. Physiol. (Lond.), 236, 549-573.

Zeki, S.M. (1991) Cerebral akinetopsia (Visual motion blindness). Brain, 114, 811-824.

Zihl, J. \& von Cramon, D.\& Mai, N. (1983) Selective disturbance of movement vision after bilateral brain damage. Brain, 106, 313-340. 